\title{
Evaluación de factores asociados a formación de colecciones intraabdominales en apendicectomía monopuerto para apendicitis aguda complicada
}

\author{
Evaluation of factors associated with formation of intra-abdominal collections in \\ single-port appendectomy for complicated acute appendicitis
}

\author{
Luis F. Cabrera ${ }^{1,2,3}$, Andrés Mendoza-Zuchini ${ }^{1,3}$, Felipe Bernal ${ }^{1,3}$, Mauricio Pedraza ${ }^{1 *}$, Juan S. Martínez ${ }^{1}$ y \\ Carlos E. Olarte ${ }^{1}$ \\ ${ }^{1}$ Departamento de Cirugía General, Universidad El Bosque; ${ }^{2}$ Departamento de Cirugía General, Fundación Santa Fe de Bogotá; ${ }^{3}$ Departamento \\ de Cirugía General, Cobos Medical Center. Bogotá, Colombia
}

\begin{abstract}
Resumen
Objetivo: Comparar los desenlaces entre la apendicectomía por laparoscopia por puerto único y multipuerto, y establecer si existe diferencia en el riesgo de colección intraabdominal posoperatoria. Método: Se realizó un estudio retrospectivo, observacional, mediante 116 historias clínicas de pacientes llevados a apendicectomía laparoscópica por único puerto y multipuerto en dos centros médicos. Resultados: Desarrollaron colección intraabdominal 12 (10.3\%) pacientes. No se encontraron diferencias entre ambas técnicas en cuanto al desarrollo de colección intraabdominal ( $p=0.242)$, no hubo diferencia entre ambas técnicas en cuanto a sangrado intraoperatorio $(p=0.012)$ y el tiempo quirúrgico fue mayor en el grupo de puerto único (17.4 minutos en promedio). El $62.5 \%$ de los pacientes con sangrado $>50 \mathrm{ml}$ desarrollaron colección intraabdominal. Conclusiones: No se evidenció superioridad de ninguna de las dos intervenciones en apendicitis complicada, pero sí se confirma que la apendicectomía laparoscópica por puerto único es un procedimiento seguro, factible, no inferior y con tasas similares de complicaciones en comparación con la técnica convencional de apendicectomía laparoscópica.
\end{abstract}

Palabras clave: Cirugía. Monopuerto. Laparoscopia. Apendicectomía.

\begin{abstract}
Objective: To compare the decreases between the appendectomy by single port vs. multiport laparoscopy and to establish if there is a difference in the risk of postoperative intra-abdominal collection. Method: Retrospective study was carried out using 116 medical records of patients undergoing laparoscopic appendectomy through a single port and multiport in two medical centers. Results: 12 (10.3\%) patients developed an abdominal collection. No differences were found between the two techniques in terms of intra-abdominal collection development $(p=0.242)$, there was no difference between the two techniques in terms of intraoperative bleeding $(p=0.012)$, the surgical time was greater in the single-port group (17.4 min on average). $62.5 \%$ of patients with bleeding $>50 \mathrm{~mL}$ developed intra-abdominal collection. Conclusions: There was no evidence of superiority of either of the two interventions in complicated appendicitis, but it does confirm that single-port laparoscopic appendectomy is a safe, feasible, noninferior procedure and with similar complication rates compared to the conventional laparoscopic appendectomy technique.
\end{abstract}

Key words: Surgery. Single port. Laparoscopic. Appendectomy.

\section{Correspondencia:}

*Mauricio Pedraza

Carrera 72, 81-55

Fecha de recepción: 11-05-2020

C.P. 111166, Bogotá, D.C., Colombia $\quad$ Fecha de aceptación: 12-08-2020

Cir Cir. 2021;89(3):384-389

E-mail: mpedraza93@gmail.com

DOI: $10.24875 / \mathrm{CIRU} .20000475$

Contents available at PubMed

www.cirugiaycirujanos.com

0009-7411/@ 2020 Academia Mexicana de Cirugía. Publicado por Permanyer. Este es un artículo open access bajo la licencia CC BY-NC-ND (http://creativecommons.org/licenses/by-nc-nd/4.0/). 


\section{Introducción}

La incidencia actual de apendicitis en Europa y América es de alrededor de 100 por 100,000 personas por año'. La apendicectomía es uno de los procedimientos quirúrgicos más frecuentes ${ }^{2}$. Este procedimiento se ha realizado durante más de 100 años, después de que McBurney lo describiera por primera vez ${ }^{3}$. Actualmente, al revisar la literatura encontramos que el método de referencia para el tratamiento de la apendicitis aguda es la apendicectomía laparoscópica por multipuerto o convencional (tres puertos). La apendicectomía laparoscópica convencional es un procedimiento seguro y factible. Sus beneficios propuestos incluyen una estancia hospitalaria posoperatoria reducida, un mejor control del dolor posoperatorio y un retorno más rápido a la actividad diaria, así como mejores resultados cosméticos, en comparación con la apendicectomía abierta ${ }^{4}$.

Con los avances tecnológicos, los cirujanos han desarrollado procedimientos mínimamente invasivos que proporcionan seguridad y beneficios para el paciente. Se ha descrito la utilización de orificios naturales (NOTES, Natural Orifice Transluminal Endoscopic Surgery) y apendicectomías por puerto único, también llamada cirugía endoscópica transumbilical de orificio natural embrionario (E-NOTES), en las que se utiliza la cicatriz embrionaria para introducir por este reparo anatómico un puerto único, utilizando los equipos convencionales de laparoscopia, y de esta manera realizar el procedimiento quirúrgico ${ }^{5-7}$. Un desarrollo reciente en la apendicectomía ha sido la introducción de la cirugía laparoscópica de incisión única menos invasiva, que utiliza un único puerto multiluminal o múltiples puertos monoluminales, a través de una única incisión en la pie|8,9.

El abordaje por puerto único en algunos de los estudios realizados ha demostrado ser igual de seguro que la apendicectomía por multipuerto, e incluso con mejores resultados cosméticos, mejor control del dolor, menor tasa de infección y mayor satisfacción por parte del paciente, sin aumentar los costos, los tiempos quirúrgicos ni el riesgo de complicaciones ${ }^{9,10}$. Aún existe gran controversia por parte algunos cirujanos, que no ven como una buena alternativa esta opción de manejo, ya que implica menor ergonomía, mayor destreza y más tiempo para alcanzar la curva de aprendizaje. Sin embargo, al revisar la literatura son pocos los estudios observacionales controlados que comparen estos dos abordajes en pacientes con apendicectomía complicada, y aun con realización de procedimientos de mayor complejidad, como cecectomía por puerto único.

Se realizó un estudio retrospectivo con el fin de comparar, con el manejo laparoscópico convencional, la eficacia y la seguridad de la técnica por puerto único, sin que se afecten los costos ni los tiempos quirúrgicos, en apendicitis complicada e incluso requiriendo intervenciones de mayor complejidad, como la cecectomía, evaluando el riesgo de colección intraabdominal.

\section{Método}

\section{Población y muestra}

Se estableció la prevalencia de colección intraabdominal en pacientes con apendicitis aguda complicada intervenidos quirúrgicamente por laparoscopia, por puerto único y multipuerto, la cual fue del $10.3 \%$. Posteriormente se determinó el tamaño de la muestra, que se definió con la utilización del software Epidat y que arrojó una muestra de 114 pacientes con un margen de error del $3 \%$.

Fueron intervenidos 116 pacientes con similares características demográficas. Los procedimientos fueron realizados por tres cirujanos con la misma formación académica. Se obtuvieron los datos de manera retrospectiva de los 116 pacientes; de ellos, $54(46.5 \%)$ correspondían a abordaje por técnica laparoscópica por multipuerto y $62(53.5 \%)$ a abordaje laparoscópico por puerto único. Las intervenciones por abordaje con puerto único se realizaron por vía transumbilical con un puerto no convencional con guante de látex (glove port); se utilizaron tres trócares a través del puerto previamente acoplado a un dispositivo de cirugía laparoscópica (Alexis). Todos los pacientes recibieron antibióticos intravenosos como mínimo durante 3 días, y más en caso de que la respuesta clínica no fuera la adecuada después de este tiempo.

\section{Criterios de inclusión}

- Pacientes mayores de 15 años.

- Pacientes con apendicitis aguda complicada.

- Cirugía laparoscópica por puerto único.

- Cirugía laparoscópica convencional de tres puertos.

- Colección intraabdominal. 


\section{Criterios de exclusión}

- Apendicectomía por puerto único por laparoscopia por periapendicitis debida a enfermedad inflamatoria pélvica.

- Pacientes llevados a apendicectomía por puerto único utilizando un dispositivo de marca registrada (como SILS [single incision laparoscopic surgery] o Gelport).

- Pacientes con diagnóstico de apendicitis complicada con manejo por cirugía abierta.

- Pacientes que fueron llevados a apendicectomía de intervalo por laparoscopia por puerto único o convencional.

- Pacientes con hallazgos intraoperatorios como neoplasias apendiculares 0 carcinomatosis peritoneal.

\section{Técnica de recolección de datos}

La recolección de datos se realizó a partir de la revisión de las historias clínicas de manera directa por los investigadores, previa aprobación de las instituciones involucradas en el estudio, y se registraron de forma organizada en el formato de recolección de datos y posteriormente fueron digitalizados en una tabla de Excel para ser tabulados y analizados estadísticamente.

\section{Plan de análisis de datos}

Se utilizó el programa Statistical Package for the Social Sciences (SPSS). Las variables de tipo cualitativo se expresaron en términos de proporciones relativas y porcentajes. A las variables cuantitativas se les calculó el promedio y el rango. Se realizó la prueba de corrección por continuidad y la razón de verosimilitudes estadístico exacto de Fisher.

En todos los casos se consideró $p<0.005$ como criterio para establecer la significación estadística.

\section{Consideraciones éticas}

Los investigadores declaran que están familiarizados con las normas para investigación en seres humanos basadas en el Código de Núremberg, el Reporte Belmont y la Declaración de Helsinki. Este estudio está clasificado como investigación sin riesgo según la resolución 8430 de 1993, dado que se emplea la consulta de una base de datos y no se realiza ninguna intervención ni modificación de las variables fisiológicas, biológicas, psicológicas y sociales de los participantes del estudio. Además, la exención de algún participante no le afectará negativamente en su bienestar ni en sus derechos. El protocolo del estudio se presentó ante las instituciones médicas para su aprobación. Todos los pacientes intervenidos quirúrgicamente tienen conocimiento del procedimiento quirúrgico por medio del consentimiento informado, previamente explicado por el cirujano y firmado por el paciente.

\section{Resultados}

Se revisaron 116 historias de pacientes intervenidos quirúrgicamente por laparoscopia en dos centros hospitalarios durante el periodo comprendido desde enero de 2018 hasta marzo de 2019, de las cuales $54(46.5 \%)$ corresponden a abordaje por técnica laparoscópica por multipuerto y $62(53.5 \%)$ a abordaje laparoscópico por puerto único. La edad promedio del grupo intervenido por abordaje multipuerto fue de 31.3 años, mientras que la del grupo intervenido por abordaje único fue de 30.5 años. El índice de masa corporal promedio fue de 33.7 en el grupo multipuerto y de 32.9 en puerto único. El sangrado promedio fue de $31.31 \mathrm{ml}$ en el grupo multipuerto y de $29.2 \mathrm{ml}$ en el grupo de puerto único. El tiempo quirúrgico promedio fue de 35.2 minutos en el grupo multipuerto y de 38.3 minutos en el grupo de puerto único. En 14 (25\%) casos se practicó cecectomía (promedio de estancia hospitalaria 5.5 días en el grupo multipuerto y 5 días en el grupo de puerto único). No hubo necesidad de conversión a cirugía abierta en ninguno de los dos grupos y el $100 \%$ de los pacientes en ambos grupos tenía apendicitis aguda complicada (Tabla 1).

Desarrollaron colección intraabdominal (Infección de sitio operatorio [ISO] grado III) 12 (10.3\%) pacientes, de los cuales $4(33.3 \%)$ corresponden al abordaje por puerto único y $8(66.7 \%)$ al abordaje multipuerto (Tabla 2).

En 18 (15.5\%) pacientes se utilizó electrobisturí monopolar (Hook) (Tabla 3), de los cuales 2 (11.1\%) desarrollaron ISO grado III (11\%). En 98 (84.5\%) pacientes se utilizó sellador laparoscópico de superficie (Tabla 3), de los cuales 9 (9.1\%) presentaron infección de sitio operatorio.

En cuanto al sangrado intraoperatorio, en el grupo de monopuerto 61 (98\%) pacientes tuvieron un sangrado usual menor de $50 \mathrm{ml}$, y solo uno presentó sangrado de 50-100 ml, mientras que en el grupo multipuerto 52 (96.3\%) pacientes tuvieron un sangrado 
Tabla 1. Características demográficas, tipo de procedimiento y desenlaces en ambos grupos

\begin{tabular}{lcc}
\hline Variable & Multipuerto $(\mathbf{n}=\mathbf{5 4})$ & Monopuerto $(\mathbf{n}=\mathbf{6 2})$ \\
\hline Edad, años & $31.3(18-65)$ & $30.5(18-56)$ \\
Índice de masa corporal & $33.7(24.1-39.2)$ & $32.9(23.5-36.8)$ \\
Sangrado, ml & $35(25-90)$ & $29(27-80)$ \\
$\begin{array}{l}\text { Tiempo quirúrgico, } \\
\text { minutos }\end{array}$ & $59.6(30-75)$ & $42.2(25-60)$ \\
$\begin{array}{l}\text { Estancia hospitalaria, } \\
\text { días }\end{array}$ & $5.5(3-7)$ & $5(3-6)$ \\
Cecectomía & 9 & 5 \\
\hline
\end{tabular}

Tabla 2. Infección de sitio operatorio de grado III

\begin{tabular}{cccccc}
\hline & No & Sí & $\mathbf{p}$ & LR \\
\hline Técnica & Monopuerto & 58 & 4 & 0.242 & 0.138 \\
& Multipuerto & 46 & 8 & & \\
\hline
\end{tabular}

LR: likelihood ratio.

Tabla 3. Asociación entre el uso de dispositivo Hook o sellador laparoscópico de superficies y el desarrollo de ISO grado III

\begin{tabular}{|c|c|c|c|c|c|c|}
\hline & & \multicolumn{5}{|c|}{ Uso de Hook } \\
\hline & & No & Sí & Total & p & LR \\
\hline \multirow[t]{2}{*}{ ISO III } & No & 88 & 16 & 104 & 1.000 & 0.908 \\
\hline & Sí & 10 & 2 & 12 & & \\
\hline \multirow[t]{3}{*}{ Total } & & 98 & 18 & 116 & & \\
\hline & & \multicolumn{5}{|c|}{ Sellador laparoscópico de superficie } \\
\hline & & No & Sí & Total & p & LR \\
\hline \multirow[t]{2}{*}{ ISO III } & No & 16 & 88 & 104 & 0.591 & 0.367 \\
\hline & Sí & 3 & 9 & 12 & & \\
\hline Total & & 18 & 98 & 116 & & \\
\hline
\end{tabular}

LR: likelihood ratio.

menor de $50 \mathrm{ml}$ y solo dos pacientes presentaron sangrado de 50-100 ml; en ninguno de los dos grupos se registró ningún sangrado mayor de $100 \mathrm{ml}$ (Tabla 4). De los 12 pacientes que presentaron ISO grado III, el $100 \%$ se asociaron con algún tipo de sangrado intraoperatorio (Tabla 5), y el $62.5 \%$ de los pacientes en que se documentó un sangrado mayor de $50 \mathrm{ml} \mathrm{y} \mathrm{el}$ $6.4 \%$ de los pacientes con un sangrado menor de $50 \mathrm{ml}$ desarrollaron colección intraabdominal.

\section{Discusión}

Actualmente, la apendicectomía por laparoscopia es el tratamiento de elección para la apendicitis aguda. Según las últimas guías de Jerusalén, publicadas por Di Saverio, et al.11, para el diagnóstico y el manejo de apendicitis aguda, es el abordaje recomendado en apendicectomía no complicada y complicada en niños y en adultos ${ }^{12}$, y además hacen mención especial a la apendicectomía laparoscópica por puerto único como una técnica que ha demostrado ser segura, factible y básicamente tan efectiva como la apendicectomía laparoscópica convencional multipuerto. Los riesgos de colección intraabdominal estimados asociados a la apendicectomía por laparoscopia oscilan entre el $1.8 \%$ y el $3.4 \% 3,10,12$. En nuestro estudio, el $10.3 \%$ de los pacientes desarrollaron una infección de sitio operatorio de grado III, un $3.4 \%$ en el grupo de puerto único y un $6.8 \%$ en el grupo de apendicectomía por laparoscopia convencional, sin haber una diferencia estadísticamente significativa entre los dos grupos $(p=0.242)$ ni predisposición a desarrollar colecciones intraabdominales con una técnica u otra (Tabla 2), acorde con lo reportado por Zhou, et al. ${ }^{13}$ en su estudio.

En cuanto al uso de dispositivos para la sección del mesoapéndice, como el electrobisturí monopolar (Hook) o el sellador laparoscópico de superficies, no mostraron tener relación con el desarrollo de ISO grado III, como se muestra en la tabla 3. En un estudio llevado a cabo por Di Saverio, et al. ${ }^{12}$ no se evidencia diferencia en el desarrollo de colección intraabdominal asociada a la utilización de los diferentes dispositivos eléctricos de disección, lo cual concuerda con nuestros hallazgos, pues tampoco logramos identificar diferencias ni asociación entre ambos dispositivos en cuanto al sangrado intraoperatorio con el uso de los distintos tipos de energía. En promedio, el sangrado intraoperatorio calculado fue de $35 \mathrm{ml}$ con el abordaje laparoscópico por monopuerto y de $29 \mathrm{ml}$ con el abordaje laparoscópico multipuerto. Carter, et al. ${ }^{14}$ también compararon ambas técnicas quirúrgicas y no encontraron diferencias en cuanto a sangrado intraoperatorio u otras complicaciones; solo parecía haber un poco más dolor posoperatorio en el grupo de cirugía por puerto único. Cabe destacar que en nuestro estudio se identificó que un $62.5 \%$ de los pacientes cuyo sangrado intraoperatorio fue mayor de $50 \mathrm{ml}$ desarrollaron una colección intraabdominal, en comparación con el $6.4 \%$ de los pacientes en quienes se cuantificó el sangrado en una cantidad menor 
Tabla 4. Asociación entre sangrado y técnica

\begin{tabular}{|c|c|c|c|c|c|c|}
\hline & & \multicolumn{2}{|c|}{ Tipo de técnica } & \multirow[t]{2}{*}{ Total } & \multirow[t]{2}{*}{$p$} & \multirow[t]{2}{*}{ LR } \\
\hline & & Monopuerto & Multipuerto & & & \\
\hline \multirow[t]{3}{*}{ Sangrado } & $0-50 \mathrm{ml}$ & 61 & 52 & 113 & 0.012 & 0.017 \\
\hline & $50-100 \mathrm{ml}$ & 1 & 2 & 3 & & \\
\hline & $>100 \mathrm{ml}$ & 0 & 0 & 0 & & \\
\hline Total & & 62 & 54 & 116 & & \\
\hline
\end{tabular}

LR: likelihood ratio.

Tabla 5. Asociación de sangrado e ISO grado III

\begin{tabular}{lcccccc}
\hline & & \multicolumn{2}{c}{ ISO III } & Total & $\mathbf{p}$ & LR \\
\cline { 3 - 5 } & & No & Sín & & & \\
\hline Sangrado & $0-50 \mathrm{cc}$ & 101 & 7 & 108 & 0.001 & 0.001 \\
& $\begin{array}{c}50-100 \\
\mathrm{CC}\end{array}$ & 3 & 5 & 8 & & \\
& $>100 \mathrm{cc}$ & 0 & 0 & 0 & & \\
Total & & 104 & 12 & 116 & & \\
\hline
\end{tabular}

LR: likelihood ratio.

$(p=0.001 ;$ IC 95\% LR 0.001), sin que se documentara asociación entre el tipo de técnica quirúrgica y la aparición de sangrado intraoperatorio (Tabla 4).

Se obtuvo como resultado un aumento en el promedio del tiempo quirúrgico con el abordaje por puerto único de 17.4 minutos con respecto a la técnica convencional multipuerto (59.6 vs. 42.2 minutos), acorde con lo publicado en las recientes guías de Jerusalén ${ }^{11}$. No se evidenciaron diferencias entre ambos grupos en cuanto a la estancia hospitalaria, la cual fue de 5.5 días en promedio.

Las técnicas mínimamente invasivas han revolucionado la cirugía; sin embargo, la superioridad del acceso laparoscópico para la apendicitis complicada aún sigue siendo controvertida para algunos ${ }^{15}$. Al comparar la presentación de colección intraabdominal, 12 de 116 pacientes intervenidos por apendicitis aguda complicada desarrollaron colecciones intraabdominales, de los cuales 4 (33.3\%) eran pacientes intervenidos por abordaje laparoscópico monopuerto y $8(66.7 \%)$ eran pacientes intervenidos por abordaje convencional multipuerto. Dados los resultados, se puede concluir que no hay superioridad de ningún procedimiento quirúrgico en el abordaje de la apendicectomía complicada. Fischer, et al. ${ }^{15}$ informaron complicaciones como colección intraabdominal similares en los dos grupos (1.4 vs. $1.8 \%$ ). De igual manera, numerosos estudios comparativos, revisiones sistemáticas y metaanálisis no han encontrado diferencias significativas en la formación de colección intraabdominal posoperatoria, en concordancia con nuestro estudio ${ }^{9,10,16,17 .}$

\section{Conclusiones}

No se evidenció superioridad de ninguna de las dos intervenciones en apendicitis complicada, pero sí se confirma que la apendicectomía laparoscópica por puerto único es un procedimiento seguro, factible, no inferior y con tasas similares de complicaciones en comparación con la técnica convencional de apendicectomía laparoscópica.

\section{Agradecimientos}

Los autores agradecen a la Universidad El Bosque, y especialmente al Departamento de Cirugía General, junto a todos sus integrantes, en particular a sus docentes, quienes han velado y perseverado por impartir sus conocimientos con humildad a cada uno de sus estudiantes. También a todas aquellas personas que de una u otra forma aportaron para la elaboración de este reporte.

\section{Responsabilidades éticas}

Protección de personas y animales. Los autores declaran que para esta investigación no se han realizado experimentos en seres humanos ni en animales.

Confidencialidad de los datos. Los autores declaran que han seguido los protocolos de su centro de trabajo sobre la publicación de datos de pacientes. 
Derecho a la privacidad y consentimiento informado. Los autores han obtenido el consentimiento informado de los pacientes y/o sujetos referidos en el artículo. Este documento obra en poder del autor de correspondencia.

\section{Conflicto de intereses}

Los autores declaran no tener ningún conflicto de intereses.

\section{Bibliografía}

1. Ohmann C, Franke C, Kraemer M, Yang Q. Neues zur Epidemiologie der Akuten Appendizitis. Der Chir. 2002;73:769-76.

2. Addiss DG, Shaffer N, Fowler BS, Tauxe RV. The epidemiology of appendicitis and appendectomy in the United States. Am J Epidemiol. 1990;132:910-25.

3. Xue C, Lin B, Huang Z, Chen Z. Single-incision laparoscopic appendectomy versus conventional 3-port laparoscopic appendectomy for appendicitis: an updated meta-analysis of randomized controlled trials. Surg Today. 2015;45:1179-86.

4. Direkze NC, Hill ADK, Darzi A. The current status of laparoscopic appendicectomy. Minim Invasive Ther Allied Technol. 2008;5:41-5.

5. Moris DN, Bramis KJ, Mantonakis EI, Papalampros EL, Petrou AS, Papalampros AE. Surgery via natural orifices in human beings: yesterday, today, tomorrow. Am J Surg. 2012;204:93-102.
6. Schaefer M. Natural orifice transluminal endoscopic surgery (NOTES): implications for anesthesia. F1000 Med Rep. 2009;4:2-5.

7. Graur F, Elisei R, Al-Hajjar N. E-NOTES transumbilical laparoscopic appendectomy. Chirurgia (Bucur). 2013;108:571-5.

8. Rehman H, Rao AM, Ahmed I. Single incision versus conventional multi-incision appendicectomy for suspected appendicitis. Cochrane Database Syst Rev. 2011;(7):CD009022.

9. Aly OE, Black DH, Rehman H, Ahmed I. Single incision laparoscopic appendicectomy versus conventional three-port laparoscopic appendicectomy: a systematic review and meta-analysis. Int J Surg. 2016;35:120-8.

10. Markar SR, Karthikesalingam A, Di Franco F, Harris AM. Systematic review and meta-analysis of single-incision versus conventional multiport appendicectomy. Br J Surg. 2013;100:1709-18.

11. Di Saverio, Podda M, De Simone B, Ceresoli M, Augustin G, Gori A, et al. Diagnosis and treatment of acute appendicitis: 2020 update of the WSES Jerusalem guidelines. World J Emerg Surg. 2020;15:27.

12. Di Saverio, Birindelli A, Kelly MD, Catena F, Weber DG, Sartelli M, et al. WSES Jerusalem guidelines for diagnosis and treatment of acute appendicitis. World J Emerg Surg. 2016;11:1-25.

13. Zhou H, Jin K, Zhang J, Wang W, Sun Y, Ruan C, et al. Single incision versus conventional multiport laparoscopic appendectomy: a systematic review and meta-analysis of randomized controlled trials. Dig Surg. 2014;31:384-91

14. Carter JT, Kaplan JA, Nguyen JN, Lin MY, Rogers SJ, Harris HW. A prospective, randomized controlled trial single-incision laparoscopic vs conventional 3-port laparoscopic appendectomy for treatment of acute appendicitis. J Am Coll Surg. 2014;218:950-9.

15. Fischer CP, Castaneda A, Moore F. Laparoscopic appendectomy: indications and controversies. Semin Laparosc Surg. 2002:9:32-9.

16. Deng L, Xiong J, Xia Q. Single-incision versus conventional three-incision laparoscopic appendectomy: a meta-analysis of randomized controlled trials. J Evid Based Med. 2017;10:196-206.

17. Sateesh S, Subraj H, Mahesh G, Rao P. Comparative analysis between single incision and conventional laparoscopic appendectomy for acute appendicitis. Int J Res Med Sci. 2014;2:1626. 